\title{
ORIGINAL ARTICLE \\ Falls in patients with HTLV-I-associated myelopathy/tropical spastic paraparesis (HAM/TSP)
}

\author{
LD Facchinetti ${ }^{1}$, AQ Araújo ${ }^{2}$, GL Chequer ${ }^{1}$, MF de Azevedo ${ }^{1}$, RVC de Oliveira $^{3}$ and MA Lima ${ }^{2}$
}

Study design: Cross-sectional study.

Objectives: To determine the prevalence of falls in human T-cell lymphotropic virus type I (HTLV-I)-associated myelopathy/tropical spastic paraparesis (HAM/TSP) patients and possible factors associated to their occurrence.

Setting: Instituto de Pesquisa Clínica Evandro Chagas, Fundação Oswaldo Cruz (FIOCRUZ) - Brazil.

Methods: Thirty-six HAM/TSP patients able to walk at least $20 \mathrm{~m}$ were assessed by a questionnaire. Data regarding gender, age, duration of disease (DD), HTLV-I proviral load (HPL), frequency of physical activity (FCA), use of walking aids, functional ambulation level, the number of falls and associated injuries in the last year were reviewed. Multiple correspondence analysis was used to group characteristics of this sample according to the fall occurrence.

Results: The prevalence of falls was $63.9 \%$ and we observed injuries in $47.8 \%$ of the cases. Four groups were identified in the descriptive analysis. One group was formed by faller individuals, men $<60$ years, independent ambulation, FCA $\geqslant 3$ times per week and HPL <6.6 copies per 100 cells (group B). The other one comprised non-faller patients, women $\geqslant 60$ years, restricted ambulation, DD $\geqslant 7$ years, use of orthosis, FCA $0-1$ time per week and HPL $\geqslant 6.6$ copies per 100 cells (group D). The others two groups comprised individuals that did not use orthosis (group A) and those that FCA was two times per week and DD $<7$ years (group C).

Conclusion: Falls occur in roughly two-thirds of ambulatory HAM/TSP patients and are associated with significant morbidity. Further studies with a larger number of patients are necessarily to identify risk factors in order to elaborate specific programs to prevent falls in this population.

Spinal Cord (2013) 51, 222-225; doi:10.1038/sc.2012.134; published online 20 November 2012

Keywords: HTLV-I-associated myelopathy; tropical spastic paraparesis; fall risk

\section{INTRODUCTION}

Human T-cell lymphotropic virus type I (HTLV-I) is a retrovirus that vary in prevalence across different geographic regions, ${ }^{1}$ being more prevalent in southwest of Japan; Caribbean; Central and South America; Central and West Africa and Melanesia. The nationwide prevalence of HTLV-I infection in Brazil is $0.45 \%{ }^{2}$

The HTLV-I can cause several immune-mediated diseases of the nervous system in which the most common is the HTLV-I-associated myelopathy/tropical spastic paraparesis (HAM/TSP). In Brazil, it is a frequent etiology of progressive myelopathy and HTLV-I is present in up to $40 \%$ of patients with chronic myelopathy. ${ }^{3}$ It manifests clinically by progressive spastic paraparesis, bladder disturbances, constipation and less conspicious sensory signs. According to Franzoi and Araújo ${ }^{4}$ about $83 \%$ of the HAM/TSP patients have sufficient function for ambulation. However, strength, age, low-back pain, duration of disease, asymmetric onset of the symptoms and spasticity may affect the ability to walk. ${ }^{5}$ All these factors could result in an impaired gait and consequently, in falls. Indeed, HAM/TSP patients frequently report unexplained falls as an early manifestation of the disease, antedating more specific symptoms of myelopathy such as lower limb weakness or sphincteric disturbances.

A fall could be defined as an unexpected event in which participants come to rest on the ground, floor or lower level. ${ }^{6}$ Similar to other neurological diseases, ${ }^{7-10}$ falls and the injuries related to them are also observed in HAM/TSP patients. Although falls are frequently reported by these patients, the risk of falls is not known in this population. Therefore, we sought to identify the prevalence of falls in HAM/TSP patients and the possible factors associated to their occurrence.

\section{MATERIALS AND METHODS}

This cross-sectional study was performed at the Instituto de Pesquisa Clínica Evandro Chagas, Fundação Oswaldo Cruz (FIOCRUZ), Rio de Janeiro, Brazil from July 2009 to March 2010. It was approved by the ethical committee and all the patients signed the informed consent. Only the patients who fulfilled the HAM/TSP criteria proposed by the World Health Organization ${ }^{11}$ and were able to walk at least $20 \mathrm{~m}$ with or without support were included in the survey. The exclusion criteria were co-infected with human immunodeficiency

${ }^{1}$ Section of Rehabilitation, Instituto de Pesquisa Clínica Evandro Chagas/Fundação Oswaldo Cruz (FIOCRUZ), Rio de Janeiro, Brazil; ${ }^{2}$ Laboratory of Clinical Research in Neuroinfections, Instituto de Pesquisa Clínica Evandro Chagas/Fundação Oswaldo Cruz (FIOCRUZ), Rio de Janeiro, Brazil and ${ }^{3}$ Laboratory of Clinical Epidemiology, Instituto de Pesquisa Clínica Evandro Chagas/Fundação Oswaldo Cruz (FIOCRUZ), Rio de Janeiro, Brazil

Correspondence: Dr LD Facchinetti, Section of Rehabilitation, Instituto de Pesquisa Clínica Evandro Chagas/Fundação Oswaldo Cruz (FIOCRUZ), Avenida Brasil 4365, Manguinhos, Rio de Janeiro 21045-900, Brazil.

E-mail: livia.dumont@ipec.fiocruz.br

Received 13 February 2012; revised 17 August 2012; accepted 2 October 2012; published online 20 November 2012 
virus (HIV); diagnosis of other neurological diseases; previous orthopedic surgeries or diseases and cognitive impairment.

After the neurological consultation in the HAM/TSP outpatient clinic, a patient completed a questionnaire under the supervision of a trained physical therapist. The information collected included demographic data, duration of disease, HTLV-I proviral load in peripheral blood mononuclear cells, practice of exercise, the use of walking aids, the number of falls and the occurrence of injuries related to falls in the last year. The Functional Ambulation Classification Scale $(\mathrm{FACS})^{12}$ was also applied. This scale is used to determine the different levels of walking ability $(0=$ non ambulation; $1=$ non functional ambulation; 2 =household ambulation; $3=$ neighborhood ambulation; $4=$ independent community ambulation; $5=$ normal ambulation). As all HAM/TSP patients had paraparesis and should be able to walk at least $20 \mathrm{~m}$ with or without support, no one was classified into the 'non ambulation' and 'normal ambulation' categories. The 'non functional ambulation', 'household ambulation' and 'neighborhood ambulation' groups were resumed in the 'Restricted ambulation' group.

Descriptive analyses were performed with the Statistical Package for the Social Science - SPSS version 16.0. Standard deviations, mean and median were used for continuous variables (age, HTLV-I proviral load and duration of disease) and frequencies for categorical variables (demographic, clinic and functional data).

We used the median values of the duration of disease and HTLV-I proviral load to create subgroups for the analysis.

Multiple correspondence analysis ${ }^{13}$ was used to group characteristics of this sample according to fall occurrence. This descriptive analysis strategy was used because it is a statistical visualization method for picturing the associations between the levels of many categorical variables. The goal of this technique is to have a global view of the data that is useful for interpretation. It is based on the interpretation of geometric distances plotted, where each point represents the level of the variables analyzed, being interpreted as associations. The graphical display facilitates the intuitive understanding of the relationships among the categories of the variables. Each of the groups indicated by the technique represents a part of the sample with common characteristics. This analysis is useful for exploratory study of risk factors, as well as in the identification of groups with similar characteristics. The advantage is not to suppose about the distribution of the variables, besides its easy interpretation. The inertias (explicative power) were used to define the number of dimensions employed in the graphic visualization of the correspondence analysis. The cluster analysis used the coordinates of the correspondence analysis in order to confirm the groups presented in the graphic. This analysis was performed with the package $\mathrm{ca}^{14}$ in $\mathrm{R}$ program version 2.13 .

Finally, the faller and non-faller groups were compared descriptively on the basis of correspondence analysis.

\section{RESULTS}

Sixty HAM/TSP patients were assessed for interview, but only thirtysix met the eligibility criteria. The median age was 51.5 (21-77) years; the duration of disease was 7 (2-28) years and HTLV-I proviral load was $6.6(0.7-54.2$ copies per 100 cells). Women represented $69.4 \%$ of the sample. About $58 \%$ of the patients practiced regular physical activity, including physical therapy.

In the functional assessment, we observed that $63.9 \%$ presented at least one fall in the last year and $47.2 \%$ of these patients had two or more falls. Injuries were observed in $47.8 \%$. The majority of the patients had an independent community ambulation (52.8\%) and used at least one orthosis (75\%).

Figure 1 demonstrates the result of the multiple correspondence analysis. We were able to select four groups of variables by visual inspection with an explicative power of variability of the sample of $68.6 \%$ in two dimensions. These groups were named as: group A those that do not use any orthosis; group B (faller) - those that had two or more falls in the last year; those that had an independent community ambulation; men; age $<60$ years; those that practiced exercise three or more times a week and a HTLV-I proviral load $<6.6$ copies per 100 cells; group $\mathrm{C}-$ those that practiced exercise two times a week and duration of disease $<7$ years; group D (non faller) - those that did not fall or had fallen only once in the last year; women; those that used any orthosis; those that do not practiced exercise or do it once a week; age $\geqslant 60$ years; duration of disease $\geqslant 7$ years; those that had restricted ambulation and a HTLV-I proviral load $\geqslant 6.6$ copies per 100 cells. The cluster analysis confirmed the results of correspondence analysis.

According to the results of multiple correspondence analysis, a comparison of demographic, clinical and functional characteristics between faller (group B) and non-faller (group D) HAM/TSP patients was performed and the results are shown in Table 1.

\section{DISCUSSION}

In the present study, we identified that $64 \%$ of the HAM/TSP patients had at least one fall in the last year and this event could be associated with injuries in almost half of the cases. High prevalence of falls had also been described in patients with incomplete spinal cord traumatic injury (75\%), ${ }^{15}$ multiple sclerosis $(54 \%)^{10}$ and poliomyelitis $(67 \%){ }^{16}$ In all these conditions, the weakness was the principal factor mentioned to be responsible for falls.

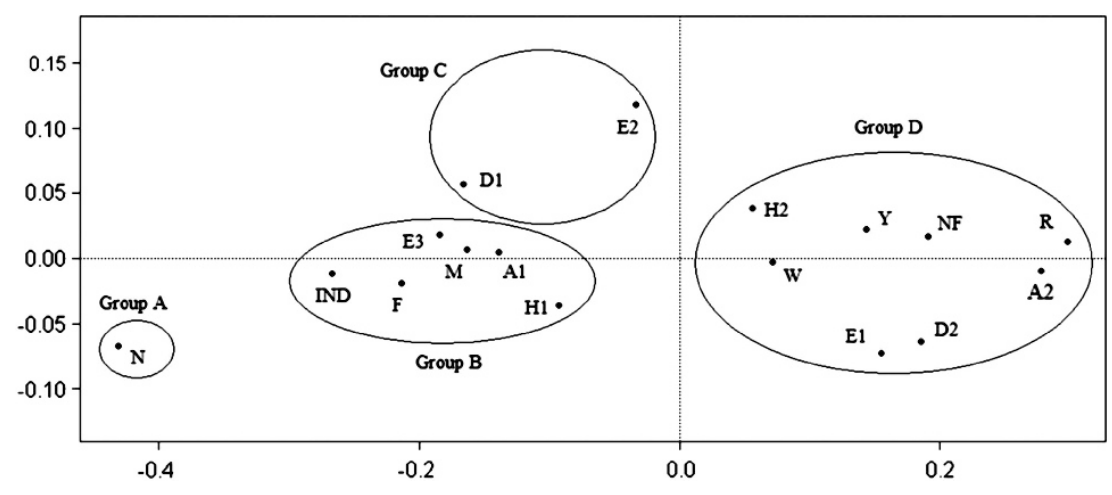

Figure 1 Graphic representation of the multiple correspondence analysis of HAM/TSP patients. M: men; W: women; A1: $<60$ years old; A2: $\geqslant 60$ years old; N: do not use orthosis; Y: use of orthosis; I: independent community ambulation in the Functional Ambulation Classification Scale; R: domiciliary or restricted community ambulation in the Functional Ambulation Classification Scale; NF: no falls or one fall in the last year; F: fallers with two or more falls in the last year; E1: do not practice exercise or do it once a week; E2: practiced exercise two times a week; E3: practiced exercise three or more times a week; D1: duration of disease <7 years; D2: duration of disease $\geqslant 7$ years; H1: HTLV-I proviral load <6.6 copies per 100 cells; H2: HTLV-I proviral load $\geqslant 6.6$ copies per 100 cells. 
Table 1 Comparison of demographic, clinical and functional characteristics between faller and non-faller HAM/TSP patients

\begin{tabular}{|c|c|c|}
\hline Characteristics & $\begin{array}{l}\text { Non-fallers (0-1 fall), } \\
\qquad n=19\end{array}$ & $\begin{array}{c}\text { Fallers }(\geqslant 2 \text { falls }), \\
n=17\end{array}$ \\
\hline \multicolumn{3}{|l|}{ Demographics } \\
\hline Age, years $(n=36)$ & $58.0(34-77)^{a}$ & $50.0(21-73)^{a}$ \\
\hline$<60$ years & $45.8 \%$ & $54.2 \%$ \\
\hline$\geqslant 60$ years & $66.7 \%$ & $33.3 \%$ \\
\hline \multicolumn{3}{|l|}{ Gender $(n=36)$} \\
\hline Female & $56.0 \%$ & $44.0 \%$ \\
\hline Male & $45.5 \%$ & $54.5 \%$ \\
\hline \multicolumn{3}{|l|}{ Clinical } \\
\hline Duration of disease, years $(n=36)$ & $11.0(2-28)^{\mathrm{a}}$ & $6.0(2-24)^{a}$ \\
\hline$<7$ years & $29.4 \%$ & $70.6 \%$ \\
\hline$\geqslant 7$ years & $73.7 \%$ & $26.3 \%$ \\
\hline $\begin{array}{l}\text { HTLV-I proviral load, copies per } 100 \\
\text { cells }(n=35)\end{array}$ & $7.0(0.7-54.2)^{\mathrm{a}}$ & $5.2(2.2-19.7)^{a}$ \\
\hline$<6.6$ copies per 100 cells & $47.1 \%$ & $52.9 \%$ \\
\hline$\geqslant 6.6$ copies per 100 cells & $55.5 \%$ & $44.5 \%$ \\
\hline \multicolumn{3}{|l|}{ Physical activity $(n=36)$} \\
\hline No +1 time per week & $43.7 \%$ & $56.3 \%$ \\
\hline 2 times per week & $50.0 \%$ & $50.0 \%$ \\
\hline$\geqslant 3$ times per week & $66.7 \%$ & $33.3 \%$ \\
\hline \multicolumn{3}{|l|}{ Functional } \\
\hline \multicolumn{3}{|l|}{ FACS $(n=36)$} \\
\hline Restricted group ${ }^{\mathrm{b}}$ & $70.6 \%$ & $29.4 \%$ \\
\hline Independent community group & $36.8 \%$ & $63.2 \%$ \\
\hline \multicolumn{3}{|l|}{ Orthosis $(n=36)$} \\
\hline No & $22.2 \%$ & $77.8 \%$ \\
\hline Yes & $63.0 \%$ & $37.0 \%$ \\
\hline
\end{tabular}

Abbreviations: HAM/TSP, HTLV-I-associated myelopathy/tropical spastic paraparesis; FACS, functional ambulation classification scale.

Indicates the values expressed in median and range.

'The 'non functional ambulation', 'household ambulation' and 'neighborhood ambulation' groups were resumed in the 'Restricted ambulation' group.

Considering that the HAM/TSP is a very disabling condition, ${ }^{17}$ only $58 \%$ of the patients were engaged in regular physical activities. Because of the variable prevalence of the HAM/TSP around the world, many health professionals are not aware of the pathophysiology or the clinical course of the disease. This scenario results in pitfalls in the treatment and the prescription of exercises that do not necessarily attenuate the progression of the disease or prevent complications such as contractures.

In the faller group, the walking ability seems to be the determining factor for the occurrence of falls in HAM/TSP patients. As observed in Figure 1, the recurrent fallers (two or more falls) practiced exercise three or more times a week and presented an independent community ambulation. These patients are able to walk in all types of irregular surfaces, including steps, stairs, ramps and curbs. ${ }^{12}$ Therefore, the mobility is not a great problem, allowing the individuals to participate in rehabilitation programs and others physical activities. However, the patients in this condition are exposed to environmental hazards, which can compromises the balance. Age $<60$ years seemed to predispose the individuals to unrestricted walking distance.

The non-faller group was represented by the individuals that used orthosis, had a higher HTLV-I proviral load and the duration of disease $\geqslant 7$ years. All these characteristics would suggest that these individuals are more disabled. Nevertheless, it is possible that the use of additional support could represent a protective factor in prevention to falls in HAM/TSP patients, such as observed by Brotherton et al. ${ }^{18}$ in individuals with incomplete spinal cord injury. Besides the use of orthosis, the long-time experience with a poor functional condition also contributes to prevent falls. Probably, it makes the HAM/TSP patient to know your limitations and adopt strategies that avoid risk situations. The non-faller group was also represented by the patients that had a restricted ambulation, the age $\geqslant 60$ years old and those that do not practice exercise or do it once a week. A worse functional status and predisposition of elderly people to falls probably contributed to limit the mobility in these individuals. This scenario was possibly difficult to the participation in community activities, corroborating the low frequency of physical activities.

Considering that the mean age of the HAM/TSP individuals is about 50 years, elderly patients are not rare in this population. We could speculate that age would be one of the main causes of falls in our sample. However, it is important to note that the proportion of individuals with $\geqslant 60$ years in the faller group was only $\sim 30 \%$. Hence, the high risk of falls in HAM/TSP individuals cannot be attributed to the advanced age, but probably to the neurological disabilities associated to the HAM/TSP.

There are some limitations in this study. First, the small size of the sample did not allow comparative analyses and, consequently, inferences to HAM/TSP population. Second, the patients were not asked to specify the physical activity practiced, maybe resulting, in some cases, in an erroneous classification. Last, the retrospective acquisition of the number of falls in the last year could be yielded to memory bias, especially in elderly individuals.

In conclusion, falls occurred in roughly two-thirds of ambulatory HAM/TSP patients and are associated with significant morbidity. Independent community ambulation, male gender, age under 60 years, low HTLV-I proviral load and practice of exercise three or more times a week were the characteristics more prevalent in patients with a history of falls. Further studies with a larger number of patients are necessary to identify the risk factors to falls in HAM/TSP patients and to develop specific programs to prevent falls in this population.

\section{DATA ARCHIVING}

There were no data to deposit.

\section{CONFLICT OF INTEREST}

The authors declare no conflict of interest.

\section{ACKNOWLEDGEMENTS}

Conselho Nacional de Desenvolvimento Científico e Tecnológico (CNPq).

1 Hela C, Shepperd S, Khumalo NP, Taylor GP. The prevalence of human T-cell lymphotropic virus type 1 in the general population is unknown. AIDS Rev 2009, Oct-Dec11: 205-214

2 Araujo AQ, de Andrada-Serpa MJ. Tropical spastic paraparesis/HTLV-Iassociated myelopathy in Brazil. J Acquir Immune Defic Syndr Hum Retrovirol 1996; 13(Suppl 1): S33-S37.

3 Figueiroa FL, Andrade FAS, Crvalho ES, Brites C, Badaro R. HTLV-I associated myelopathy: clinical and epidemiological profile. Braz J Infect Dis 2000; 4: 126-130.

4 Franzoi AC, Araujo AQ. Disability profile of patients with HTLV-I-associated myelopathy/ tropical spastic paraparesis using the functional independence measure (FIM). Spinal Cord 2005; 43: 236-240

5 Franzoi $A C$, Araujo AQ. Disability and determinants of gait performance in tropical spastic paraparesis/HTLV-I associated myelopathy (HAM/TSP). Spinal Cord 2007; 45: 64-68. 
6 Lamb SE, Jorstad-Stein EC, Hauer K, Becker C. Development of a common outcome data set for fall injury prevention trials: the prevention of falls network europe consensus. J Am Geriatr Soc 2005; 53: 1618-1622.

7 Tinetti ME, Speechley M, Ginter SF. Risk factors for falls among elderly persons living in the community. N Engl J Med 1988; 319: 1701-1707.

8 Dibble LE, Lange M. Predicting falls in individuals with Parkinson disease: a reconsideration of clinical balance measures. J Neurol Phys Ther 2006; 30 : 60-67.

9 Wallace C, Reiber GE, LeMaster J, Smith DG, Sullivan K, Hayes S et al. Incidence of falls, risk factors for falls, and fall-related fractures in individuals with diabetes and a prior foot ulcer. Diabetes Care 2002; 25: 1983-1986.

10 Cattaneo D, De Nuzzo C, Fascia T, Macalli M, Pisoni I, Cardini R. Risks of falls in subjects with multiple sclerosis. Arch Phys Med Rehabil 2002; 83: 864-867.

11 Osame M. Human Retrovirology: HLTV. In: Blattner WA (ed.), 1st edn. Raven Press: New York, 1990: 191-197.
12 Viosca E, Martinez JL, Almagro PL, Gracia A, Gonzalez C. Proposal and validation of a new functional ambulation classification scale for clinical use. Arch Phys Med Rehabil 2005; 86: 1234-1238.

13 Nenadic O. GM. correspondence analysis in R, with two- and three-dimensional graphics: the ca package. J Stat Softw 2007; 20: 1-13.

14 Greenacre MJ (ed.) Correspondence Analysis in Practice, 2nd edn. Chapman \& Hall/ CRC: London, 2007.

15 Brotherton SS, Krause JS, Nietert PJ. Falls in individuals with incomplete spinal cord injury. Spinal Cord 2007; 45: 37-40.

16 Lord SR, Allen GM, Williams P, Gandevia SC. Risk of falling: predictors based on reduced strength in persons previously affected by polio. Arch Phys Med Rehabil 2002; 83: 757-763.

17 Olindo S, Cabre P, Lezin A, Merle H, Saint-Vil M, Signate A et al. Natural history of human T-lymphotropic virus 1-associated myelopathy: a 14-year follow-up study. Arch Neurol 2006; 63: 1560-1566.

18 Brotherton SS, Krause JS, Nietert PJ. A pilot study of factors associated with falls in individuals with incomplete spinal cord injury. J Spinal Cord Med 2007; 30: 243-250. 